\title{
GASTRIC BEZOAR - A RARE PRESENTATION IN INFANT
}

\author{
MTH SIDDIQUI, MM ALAM, AM SHAHINUR, AZM ZAHID, GZ HASAN
}

\begin{abstract}
:
Gastric bezoar in neonate is a rare presentation. Because of rarity of phytobezoar and lactobezoar are not considered as the differential diagnosis of a lump and vomiting commonly. A 7 month female presented with left upper abdominal undifferentiated hard mobile lump and post prandial occasional vomiting. Diagnosis was non-conclusive with USG, contrast medium study of stomach and duodenum and contrast enhanced CT scan. After laparotomy and gastrostomy mass was removed and diagnosed as phytolactobezoar on macroscopic appearance
\end{abstract}

Key word: Phytolactobezoar, infant.

\section{Introduction:}

Retained concretion of indigestible foreign materials that accumulate and conglomerate in the gastrointestinal tract are known as bezoar. Overtime they associate with mucous and form masses. Most commonly they are found in the stomach but can be found anywhere from the esophagus to the rectum. Phytobezoars are the most common type of bezoars today, those composed of nondigestible food materials by humans including cellulose, hemicellulose, lignin, and fruit tannins. (Holloway WD 1980, Matsuo T 1978) Lactobezoars are a compact mass of undigested milk concretions in the gastrointestinal tract, traditionaly associated with preterm infant fed a highly

1. Dr M T H Siddiqui, Professor, Department of Pediatric Surgery, BSMMU.

2. Dr Mohammad Mahabubul Alam, Resident, Phase-B, Department of Pediatric Surgery, BSMMU.

3. Dr A M Shahinur, Medical Officer, Department of Pediatric Surgery, BSMMU.

4. DrAK M Zahid Hossain, Associate Professor, Department of Pediatric Surgery, BSMMU.

5. Dr. Gazi Zahirul Hasan, Associate Professor, Department of Pediatric Surgery, BSMMU.

Correspondence to : Dr M T H Siddiqui, Professor, Department of Pediatric Surgery, BSMMU, E-mail: t_siddiqui_2000@yahoo.com concentrated formula in the first few weeks of life (Sanders MK 2004). The critical elements in the diagnosis, management prevention of gastrointestinal bezoars are understanding the pathophysiology of formation and identifying high-risk individuals(Sanders MK 2004). Recent years, bezoars are considered as a potentially serious medical problem in patients with compromised gastric anatomy and/or gastrointestinal motility. First report of human trichobezoar was made by Baudamant in 1779. Most of the articles on bezoars are case reports or case series, with only a few on pediatric cases. Among pediatric case report and case series bezoars are reported as a rarer case in infant. We have encountered one infant (7 months old) of primary stomach Phytolactobezoar who presented with undifferentiated upper abdominal lump. Due to rarity of Phytobezoar was usually not considered as a differential diagnosis. This case is reported due to extreme age.

\section{Case report}

A 7 months old girl, presented with 20 days history of undifferentiated abdominal lump in left upper quadrant of abdomen with post prandial non-projectile, nonbilious vomiting. She was on exclusive breast feeding during first 2 months of her age followed byformula feed and mashed banana. There was no constipation or urinary complaints. Her past history revealed that she suffered from acute gastroenteritis which was managed after hospitalization. Her birth history was uneventful and developmental milestones are normal. She was fully immunized as per EPI (Expanded Program on Immunization)schedule. 
On examination she looked ill, mild pale, anicteric. Her body weight and length were normal for her age and sex. Vital parameters were within normal limit. The abdomen was soft, with a large nontender, noncompressible firm mass in the left side of abdomen in theleft hypochondria extending to epigastrium and umbilical area. The mass was rounded borders, smooth surface and moved in all directions. Bowel sounds were active. No organomegaly was noted. Other systemic examination was unremarkable. With a working diagnosis of undifferentiated upper abdominal mass laboratory investigation and imaging was done. Her hemoglobin was $11.1 \mathrm{gm} / \mathrm{dl}$. Leukocyte count 16.0/cumm, platelet count and differential count of WBCs were normal. Serum electrolytes and serum creatinine $(0.45 \mathrm{mg} / \mathrm{dl})$ are within normal limit. Plain abdominal X-ray demonstrated a soft tissue mass occupying central upper abdomen with extension to the right side with no evidence of calcification. Ultrasonography of whole abdomen showed a hyper echoic mass casting acoustic shadow in the epigastric region.

Contrast medium study of stomach and duodenum with fluoroscopy and spot radiography showed large doubtful filling defect within the fundus and body of distended stomach. No outlet obstruction seen. [Fig; 1]

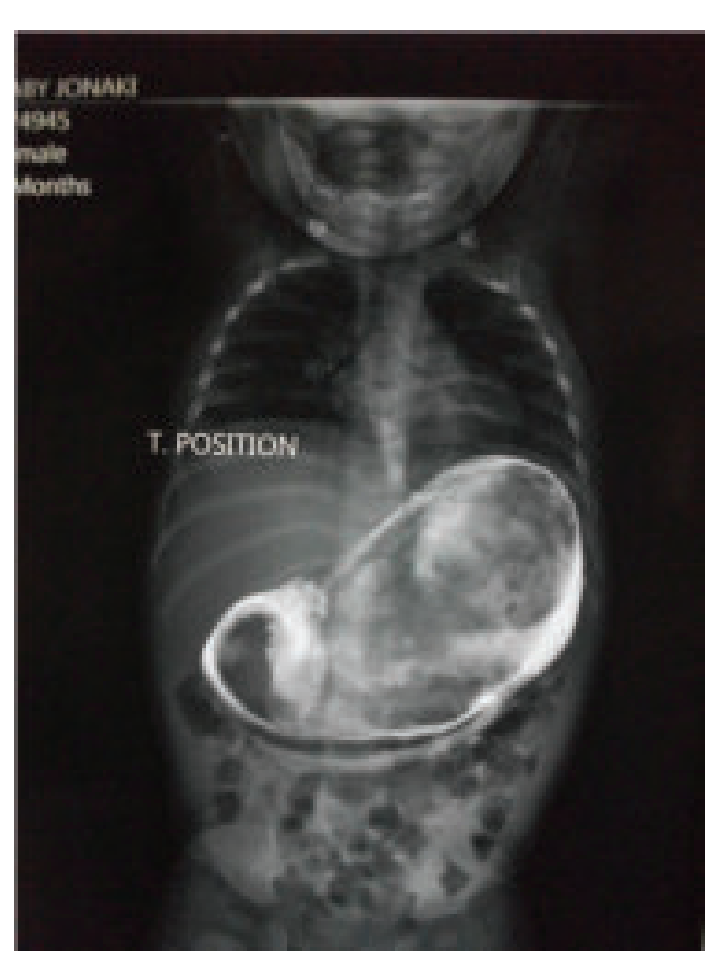

Fig.-1: Contrast medium study of stomach and duodenum shows doughtful feeling defect
Contrast enhanced (both oral \& I.V) CT scan of abdomen showed distention of stomach with much delayed gastric emptying. There found heterogeneous dense mass showing "rail track" appearance within it. Duodenum is normally formed and small amount of contrast medium are noted within coils of small intestine which are dilated due to pressure effect of the mass. [Fig. 2]

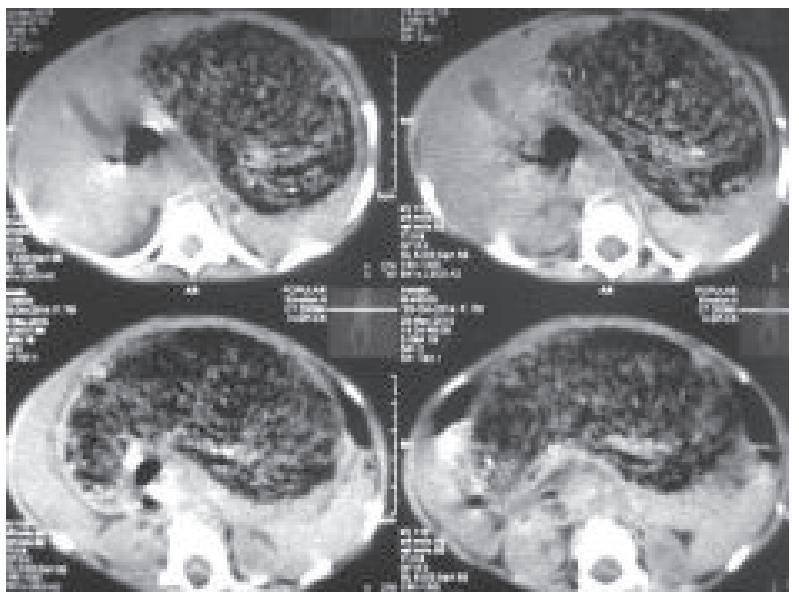

Fig.-2: Contrast enhanced CT scan of abdomen shows gastric lactophytobezoar

Due to rarity of the cases radiologists of a peripheral medical college could not formulate a diagnosis. They suspected some differentials of the condition as neuro-ectodermal mass lesion of stomach, multiple round worm within stomach or dermoid. Baby was referred to Bangabandhu Sheikh Mujib Medical University Hospital and total history, physical findings and images with laboratory data were reevaluated. Although very rare but according to feeding history and image findings bezoar was suspected. Exploratory laparotomy was done through an upper abdominal transverse incision. Stomach was full with a smooth solid stomach shaped mass freely moved when pressed from the antrum. The stomach shaped black to brown hard block of phytolactobezoar was identified and retrieved after anterior longitudinal gastrotomy. [Fig 3,4,5] Pylorus, duodenum and small intestine were checked for luminal patency and found ok. Gastrostomy and laparotomy wound was closed in layers. She had a good post-operative recovery.

Vomitus stopped and wound healed as expected. Advice for feeding according to her age and socioeconomic condition was given. Schedule for follow-up visit was given. 


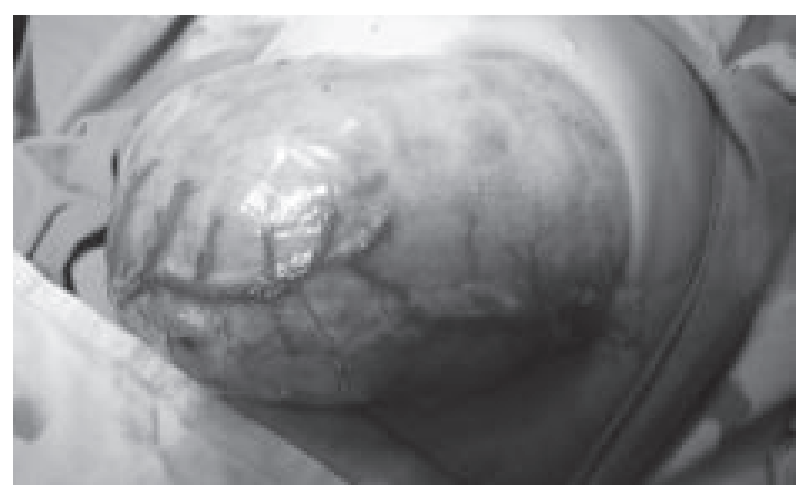

Fig.-3: Stomach after laparotomy

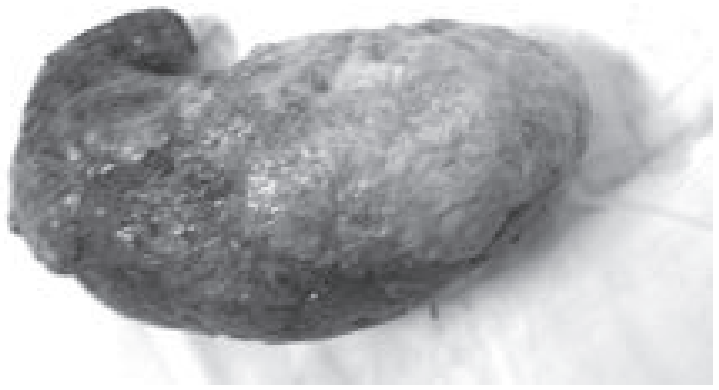

Fig.-5: Phytolactobezoar removed after gastrotomy

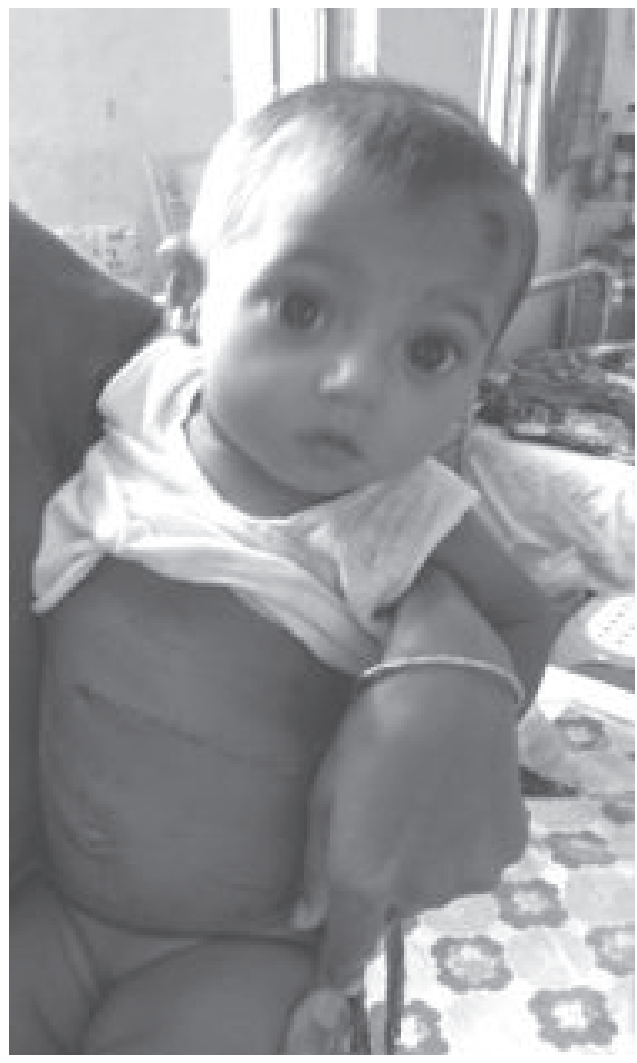

Fig.-6: During the day of discharge

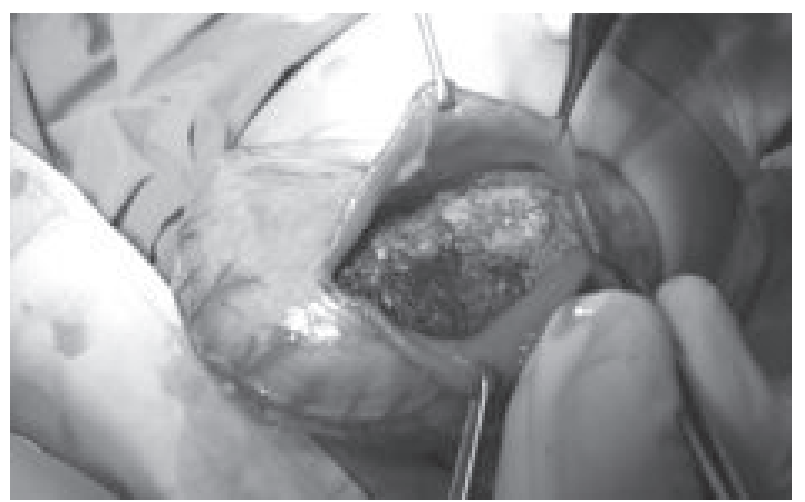

Fig.-4: Bezoar on gastrotomy

\section{Discussions:}

The term bezoar comes from either the Persian "pahnzer" or the Arabic "badzehr," both of which mean antidote or counter-poisonand have been described in human and ruminants gastrointestinal tracts for centuries (Williams RS, 1986 and Andrus CH, Ponsky $\mathrm{JL}, 1988)$.Bezoars are classified into five types on the basis of their origin and components: (1) Phytobezoar (Undigested food particles found in fruit and vegetables); (2) Trichobezoar (composed of hair); (3) Lactobezoar (compact mass of undigested milk concretions and reported only in neonate on highly concentrated formula); (4) Pharmacobezoar (conglomeration of medications like extended release products, bulk-forming laxatives); (5) Foreign body bezoar (chewngum candy, toilet paper, persimmons \& seeds) (Quercioli A, 2009).

Gastric lactobezoars (GLBs) are the most common type of bezoars found in infants[6]. They were first described in low birth weight infants in 1959 (Wolf RS, and Bruce J, 1959). Gastric bezoars may be found in asymptomatic patients but usually present with gastrointestinal symptoms, such as abdominal distension, vomiting, regurgitation, gastric residuals or palpable abdominal mass (Heinz-Erian P 2012). More rarely it can appear as gastric outlet obstruction. The pathogenesis of gastric lactobezoar includes mostly exogenous influences such formulas with high casein contents, medium chain triglyceride oils, and increased caloric density milk. Endogenous factors include an immature gastrointestinal tract and dehydration (Heinz-Erian P 2012). However, a GLB has been documented in a term, exclusively breastfed infant (Bajorek S2012). Our patient possessed some of the above risk factors including consuming a high caloric density formula with a history of ingestion of 
mashed banana. Clinicians must be cognizant that these clinical management decisions may result in the development of a gastric phytolactobezoar. Diagnosis requires a high index of suspicion and experienced investigators. Abdominal ultrasound, the method of choice, reveals highly echogenic mass. Upper gastrointestinalcontrast X-ray can be diagnostic but is inconclusive in about $30 \%$ of the cases (Sanders MK 2004). On our case, diagnosis was made solely after exploratory laparotomy, even though in review of prior exams, there was an identifiable intra-gastric mass on abdominal ultrasound, contrast medium-ray of upper gastrointestinal tract and contrast enhanced computed tomography(Heinz-Erian P 2012).

Conservative management is effective in over $85 \%$ of the cases; however, surgical measures may be necessary if the condition does not resolve within 72 $\mathrm{h}$, in case of complications or in large size bezoar (Heinz-Erian P 2012).In the limited studies report mentioned cellulose as an attractive medication with success rate $100 \%$ with no prominent adverse effect. Patients with delayed gastric emptying may be beneficial from long term therapy with prokinetic agents like metoclopramide for the management and prevention of gastric bezoar (Sanders MK 2004). There have been reports of successful disintegration of lactobezoars using intra-gastric Nacetylcysteine(Bajorek S 2012). Endoscopic therapy focuses on mechanical disruption using instruments such as tripod forceps, polypectomy snares, water piks, neodymium yttrium aluminum garate (Nd:YAG) laser (Sanders MK 2004). However surgery is the choice in case of large and late diagnosed bezoar.

\section{Conclusion:}

Intestinal phytobezoar and lactobezoar in childhood is a rare benign condition.In this case although prediction of bezoar is very unusual, but careful feeding history could raise the suspicion. Strong clinical suspicion is important in making a correct preoperative diagnosis. In such cases noninvasive method like ultrasonography, barium meal and CT scan can be of immense help.

\section{References:}

1. Andrus $\mathrm{CH}$, Ponsky JL. Bezoars. classification, pathophysiology, and treatment. Am J Gastroenterol, 1988;83(5):476-478.

2. Bajorek S, Basaldua R, McGoogan K, Miller C, Sussman C. Neonatal gastric lactobezoar: management with $\mathrm{N}$-acetylcysteine. Case Rep Pediatr 2012;2012:412412.

3. Heinz-Erian P, Gassner I, Klein-Franke A, Jud V, Trawoeger R, Niederwanger C, et al. Gastric lactobezoar - a rare disorder? Orphanet J Rare Dis 2012; 7:3.

4. Holloway WD, Lee SP, Nicholson GI. The composition and dissolution of phytobezoars. Arch Pathol Lab Med, 1980; 104: 159-161.

5. Matsuo T, Ito S. The chemical structure of kakitannin from immature fruit of the persimmon. AgricBiolChem, 1978; 126: 421- 424.

6. Martin RJ, Fanarof AA, and Walsh MC, NeonatalPerinatal Medicine, Mosby Elsevier, Philadelphia, $\mathrm{Pa}$, USA, 8th edition, 2006.

7. Quercioli A, Dallegri F, Ottonello L, Montecucco $F$ and Borgonovo $G, 2009$. Intestinal radiationinduced stricture favours small bowel obstruction by phytobezoar; report a case. Hindawi Publishing Corporation Gastroenterology Research and Practice.

8. Sanders MK. Bezoars: From Mystical Charms to Medical and Nutritional Management. Practical Gastroenterology, January 2004;13:37-50.

9. Williams RS. The fascinating history of bezoars. Med J Aust, 1986;145(11-12):613-614.

10. Wolf RS, and Bruce J, 1959. "Gastrotomy for lactobezoar in a newborn infant," The Journal of Pediatrics, vol. 54, no. 6, pp. 811-812. 